\title{
LES ÉCHELLES DE TEMPS SUR LES MARCHÉS FINANCIERS
}

\author{
Christian WALTER
}

RÉSUMÉ : La modélisation financière moderne instrumente massivement des lois d'échelle pour la formalisation des fluctuations des marchés, à travers l'usage des processus aléatoires dans les équations de comportement des cours boursiers. D'abord implicites, présentes dans le mouvement brownien mais non perçues en tant que telles, les lois d'échelle sont réapparues explicitement comme enjeu de la modélisation depuis que les autorités de tutelle des marchés financiers ont attiré l'attention des établissements bancaires sur le problème du contrôle des risques, mal quantifiés par les distributions gaussiennes classiques. On présente les éléments de ce débat, en adoptant comme fil conducteur la postérité des modèles fractals de Mandelbrot dans le sillage desquels s'inscrivent les principaux conflits de modélisation des variations boursières depuis les quarante dernières années. On montre que la question de l'existence de lois d'échelle renvoie à la question plus fondamentale de la nature du temps des marchés.

Mots-CLÉs : modélisation financière, fractales, processus de Lévy, lois stables, mémoire longue, temps intrinsèque.

ABSTRACT: Modern financial modelling massively implements scaling laws to describe market fluctuations, with the use of stochastic processes in stock market price behavior equations. Scaling laws, which already existed as an implicit part of Brownian motion although notperceived at such, habe become increasingly and explicitely important, since the financial markets regulatory authorities have drawn the attention of the banking industry to the risk control issued posed by the ineffective current valuation achieved using the classical Gaussian distribution. The différent elements surrounding this debate are developed based around Mandelbrot's fractal models, which have underlined the main modelling conflicts of the behavior of stock market prices over the last forty years. I demonstrate that the root of the problem of whether scaling laws exist is the more fundemental issue of the nature of market time.

KEYWORDS : financial modelling, fractals, Lévy processes, stable distributions, long memory, intrinsic time.

Revue de synthèse : $4^{\mathrm{e}}$ S. n ${ }^{\mathrm{o}}$ 1, janv.-mars 2001, p. 55-69. 
Zusammenfassung : Bei der heute üblichen Modellierung von finanziellen Transaktionen werden in großem Umfang Maßstabsgesetze benutzt, um Marktfluktuationen zu modellieren. Dabei werden Zufallsprozesse in den Gleichungen benutzt, die die Börsenkurse beschreiben. Die in der Brownschen Bewegung implizit vorhandenen, aber nicht als solche erkannten Maßstabsgesetze traten explizit bei der Modellbildung in Erscheinung, als die Aufsichtsbehörden über die Finanzmärkte die Aufmerksamkeit der Banken auf das Problem der Kontrolle von Risiken hinwiesen, die sich mit der klassischen Gaußverteilung nur schlecht quantifizieren ließen. Hier werden die Elemente dieser Debatte vorgestellt, wobei als Leitfaden dient, was aus den Mandelbrotschen Fraktalmodellen hervorgegangen ist, in deren Nachfolge seit den vergangenen vierzig Jahren die hauptsächlichen Konflikte bei der Modellierung von Bankvorgängen zu finden sind. Es wird gezeigt, daß die Frage nach der Existenz von Maßstabsgesetzen zu der grundsätzlicheren Frage nach dem Wesen der Zeit im Marktgeschehen führt.

STICHWÖRTER: Modellierung von Finanzmärkten, Fraktale, Lévy-Prozesse, stabile Gesetze, Langzeitgedächtnis, innere Zeit.

RIASSUNTO: La modellizzazione finanziaria moderna fa un uso massiccio delle leggi di scala per la formalizzazione dei flussi dei mercati, attraverso l'uso dei processi aleatori nelle equazioni di comportamento nei corsi della borsa. Inizialmente implicite, presenti quindi nel movimento browniano ma non percepite in quanto tali, le leggi di scala sono riapparse esplicitamente come fattore della modellizzazione da quando le autorità di tutela dei mercati finanziari hanno attratto l'attenzione degli stabilimenti bancari sul problema del controllo dei rischi, mal quantificati dalle distribuzioni gaussiane classiche. Si rappresentano gli elementi di questo dibattito prendendo come filo conduttore la posterità dei modelli frattali di Mandelbrot nella scia dei quali si iscrivono i principali conflitti di modellizzazione delle variazioni in borsa negli ultimi quarant'anni. Si dimostra quindi che la questione dell'esistenza delle leggi di scala rinvia alla questione ancor più fondamentale della natura del tempo dei mercati.

PAROLE-CHIAVE : modellizzazione finanziaria, frattali, processo di Lévy, leggi stabili, memoria lunga, tempo intrinseco.

Christian WaLter, né en 1957, est professeur au département de mathématiques de l'université d'Évry, directeur chez PricewaterhouseCoopers et coorganisateur du programme « Histoire et épistémologie de la finance » à la Maison des sciences de l'homme. Ses principaux domaines de recherche sont les applications des fractales et des processus de Lévy en finance, et l'épistémologie de la modélisation financière.

Adresse: PricewaterhouseCoopers, 32 rue Guersant, F-75017 Paris.

Courrier électronique : christian.walter@fr.pwcglobal.com 
La recherche d'invariants est souvent considérée comme l'objectif premier de l'activité scientifique. Sur les marchés boursiers, cette recherche a pris le chemin de la modélisation du processus des variations des cours cotés. Très tôt s'est posée la question de la mise en évidence de régularités empiriques, de l'obtention d'une équation robuste régissant les fluctuations boursières, résultats qui ne seraient pas dépendants de la période d'observation des marchés ni affectés par les conditions changeantes des économies. L'histoire de la finance au $\mathrm{xx}^{\mathrm{e}}$ siècle est traversée par cette recherche qui, telle une vertébration des autres domaines de la modélisation, a constitué le soubassement de l'ingénierie financière puis de l'industrie, fondements probabilistes sur lesquels se construisaient les modèles économiques théoriques et les produits financiers.

De la tentative de description analytique adéquate du comportement des marchés financiers a alors surgi la question des échelles de temps pour la mesure statistique de ces variations, accompagnée de son corollaire, celle de l'agrégation temporelle des fluctuations des cours boursiers. Les analyses statistiques usuelles des fluctuations boursières considéraient indifféremment les variations hebdomadaires, mensuelles ou trimestrielles, mais sans chercher à décrire le passage d'une échelle à l'autre, ni à examiner la pertinence d'une échelle par rapport à une autre. La question de l'agrégation temporelle des fluctuations des marchés est précisément l'étude de ce passage, celui d'une analyse en haute fréquence des marchés (variations boursières sur courte période), à une analyse en basse fréquence (variations boursières sur longue période). La courte période définit un « temps court » (ou horizon de court terme), par exemple celui de l'opérateur de salle des marchés qui intervient pour une durée de quelques minutes à quelques heures. La longue période définit un «temps long » (ou horizon de long terme), qui sera plutôt celui d'un investisseur institutionnel dont les fonds représentent des engagements à longue durée (par exemple des caisses de retraite). Un marché boursier est ainsi un lieu de rencontre de temps différents, chaque temps correspondant à des objectifs économiques spécifiques.

Benoît Mandelbrot fut le premier à aborder cette question dans les années soixante, et à chercher à détecter la présence de lois d'échelle sur les marchés. Mandelbrot cherchait à mettre en évidence des invariances par changement d'échelle (c'est-à-dire une structure fractale des marchés), ce qui permettait ensuite d'observer un marché à n'importe quelle échelle arbitraire dans la mesure où il n'existait plus de temps privilégié pour capter la structure comportementale fondamentale de ses fluctuations. Toutes les échelles de temps convenaient alors indifféremment à l'analyse statis- 
tique. L'hypothèse fractale de Mandelbrot attira l'attention sur ce problème, et induisit une prise en considération nouvelle des échelles.

Une autre réponse vit alors le jour, qui proposa une description non fractale des marchés, en posant l'existence d'échelles caractéristiques, chaque échelle de temps représentant un horizon d'investissement pour une catégorie donnée d'agents économiques. Selon cette perspective, il n'y aurait pas de raison particulière pour que les variations correspondant à l'horizon court du trader et celles correspondant à l'horizon long du gérant de portefeuille soient modélisables par la même loi de probabilité. Cette coexistence simultanée de temps spécifiques nécessite plutôt d'instrumenter une analyse multiéchelle et des marchés (le temps des traders, le temps des gestionnaires, le temps des assureurs etc.) pour ne pas perdre d'information sur le phénomène étudié. Le débat autour de la modélisation fractale des marchés se situe précisément sur ce point.

À la suite d'évolutions réglementaires récentes au sein de l'industrie financière, cette question des échelles de temps a quitté le champ restreint et confidentiel de la recherche fondamentale, dans lequel elle était longtemps restée, pour entrer dans celui de la recherche appliquée, en suscitant un intérêt nouveau qui croît de manière rapide. En effet, l'introduction de lois d'échelle est corrélative d'une modification de la perception du risque des fluctuations boursières, en raison du changement de propriétés statistiques qu'elle implique. Or, depuis quelque temps, on observe dans le champ de l'industrie financière le déploiement d'une approche institutionnelle des risques de marché, approche dite «prudentielle», dont l'objectif est de promouvoir des règles de quantification des risques universellement applicables par les établissements financiers actifs sur les marchés ${ }^{1}$. Ce souci prudentiel est étroitement corrélé avec l'augmentation extrêmement rapide des encours d'instruments financiers appelés «dérivés », dont les difficultés de contrôle et de gestion constituent une source grandissante de préoccupation pour les autorités de tutelle des bourses.

Or il est apparu que les outils et méthodes classiques, tous fondés sur l'utilisation de lois d'échelle gaussiennes, ne permettaient pas de disposer de mesures fiables pour la quantification de ces risques. Un besoin de nouvelles méthodologies et de nouveaux outils est apparu, qui a contribué à accélérer la recherche théorique sur les distributions non gaussiennes et les lois d'échelle. Ce qui a entraîné de nouveaux débats dans le champ de la modélisation financière.

On présente ici les éléments de ces débats. Le fil conducteur de l'investigation entreprise sera l'histoire de la modélisation fractale en finance et la postérité des modèles de Mandelbrot, dans le sillage desquels s'inscrivent

1. Bâle, 1994, 1996, 1998 et 1999, et Iosco, 1994. 
les principaux conflits de modélisation des variations boursières qui ont agité la théorie de la finance depuis les quarante dernières années. Afin d'éviter des redites, et en raison de la place limitée ici, on se permettra, en sollicitant son indulgence, de renvoyer le lecteur à un travail antérieur ${ }^{2}$ pour l'ensemble des aspects relatifs à l'histoire de la modélisation financière précédant l'apparition des modèles de Mandelbrot, et à un travail parallèle ${ }^{3}$ pour toutes les références détaillées concernant les résultats des recherches mentionnées sur les modèles iid- $\alpha$-stables et $H$-corrélés en finance. De la même manière, les références nombreuses aux textes de Mandelbrot ne sont pas détaillées, et l'on renvoie à son ouvrage spécifique sur la finance ${ }^{4}$ pour une bibliographie complète de ses modèles. Enfin, malgré leur importance pour le thème examiné, les aspects statistiques (estimation) et économétrique (temps discret), ne sont pas abordés $\mathrm{ici}^{5}$.

LES LOIS D’ÉCHELLE IMPLICITES DE LA MODÉLISATION FINANCIÈRE CLASSIQUE

La modélisation financière pour la représentation des variations des marchés, considère les prix et les rentabilités des différents actifs comme des variables aléatoires. La succession des cours devient alors assimilable à un processus aléatoire, dont les trajectoires temporelles représentent l'évolution de la valeur de l'actif entre deux dates. Le processus aléatoire de base choisi pour cette représentation est le mouvement brownien. Ce modèle est le «modèle standard » des fluctuations boursières.

Le mouvement brownien est un processus auto-affine à accroissements indépendants et stationnaires, de loi marginale gaussienne, qui possède des propriétés fractales désormais bien connues. En particulier, ses accroissements $\Delta X=X(t+\Delta t)-X(t)$ suivent une loi d'échelle telle que

$$
\Delta X \sim(\Delta t)^{1 / 2}
$$

Cela revient à dire que la distribution du rapport $\Delta X /(\Delta t)^{1 / 2}$ est indépendante du temps. En d'autres termes, l'ordre de grandeur de la variation d'un cours boursier (du prix d'un titre) pendant une durée donnée, est proportionnel à la racine carrée de cette durée. Cela quelle que soit l'échelle de temps (la durée) considérée. Il y a donc invariance de la loi de variation du prix par changement d'échelle : la loi de variation des prix ne dépend pas de la durée de détention du titre.

\footnotetext{
2. WALtER, 1996.

3. WALTER, 2001, avec cent références bibliographiques.

4. Mandelbrot, 1997.

5. On pourra, p. ex., consulter sur cette question GuÉGAN, 1994, ou BAILlie, 1996.
} 
Les acteurs des marchés appliquent en permanence cette propriété fractale. En finance, la rentabilité et le risque associés sont proportionnels au temps écoulé. La relation (1) donne cette proportionnalité, fonction de la valeur de l'exposant (1/2): le risque théorique d'un actif financier va se dilater en racine carrée du temps (l'exposant d'autosimilitude 1/2). Ainsi, par exemple, le risque à 12 mois sera égal au risque à 1 mois multiplié par la racine carrée de 12 . Ce calcul de risque à temps long à partir du risque à temps court est à la base des réflexions prudentielles du Comité de Bâle évoquées en introduction.

Les accroissements du mouvement brownien représentent les rentabilités (ou variations) boursières successives. L'indépendance de ces rentabilités successives revient à faire une hypothèse économique sur la transmission de l'information dans les prix et se trouve à l'origine du concept d'efficience des marchés. La stationnarité des rentabilités signifie que les caractéristiques probabilistes du marché ne changent pas, ou «pas trop », au fil du temps. La gaussienne implique une limitation des variations possibles des rentabilités (pas de krach boursier, pas de flambée haussière). On notera « iid-gaussien » de façon abrégée, (iid signifiant « indépendamment et identiquement distribuées »), ce modèle de variations boursières.

La loi de Gauss, qui est la loi marginale des accroissements du mouvement brownien, possède une propriété très intéressante : elle vérifie la stabilité par addition au sens de Paul Lévy. La stabilité au sens de Lévy exprime une invariance par produit de convolution : la somme de variables aléatoires convenablement normalisées donne une variable aléatoire de même forme que les lois initiales à l'échelle près (ainsi, la somme de gaussiennes donne une autre gaussienne). La « forme » de la loi est déterminée par un paramètre essentiel, l'exposant caractéristique noté $\alpha$, qui prend ses valeurs entre 0 et 2 . Lorsque $\alpha=2$, il s'agit de la gaussienne. Pour toutes les autres valeurs de $\alpha<2$, les distributions sont non-gaussiennes, ont une variance infinie, et présentent des queues plus étirées ajustables par des lois de Pareto. Le modèle classique des variations boursières est ainsi un modèle que l'on peut dénommer, pour plus de généralité, «iid-2-stable ».

Appliquer le modèle iid-2-stable aux fluctuations boursières revient donc à caractériser ces fluctuations par une invariance statistique d'échelle, c'est-à-dire à poser une description fractale des marchés. C'est une des raisons pour lesquelles les analyses statistiques des marchés n'ont jamais soulevé, avant les modèles de Mandelbrot, la question du choix de l'échelle : les observations trimestrielles ou mensuelles (pour la plupart) ou hebdomadaires (pour certaines) paraissaient tout aussi pertinentes les unes que les autres. 
LE COMPORTEMENT RÉEL DES MARCHÉS BOURSIERS

ET LES MODÈLES DE MANDELBROT

Par rapport à cette modélisation iid-2-stable, un certain nombre d'anomalies sont apparues assez rapidement au cours des investigations statistiques effectuées sur les marchés boursiers. Elles ont concerné tout d'abord la question de la normalité des distributions empiriques des rentabilités, puis dans un second temps sont apparues d'autres problèmes sur les phénomènes de dépendance complexe des variations boursières successives.

\section{a) Le problème leptokurtique et le premier modèle de Mandelbrot}

Il était connu depuis déjà un certain temps que les distributions réelles observées sur les marchés présentaient un trop grand nombre de grandes valeurs (fortes rentabilités positives ou négatives) pour pouvoir être ajustées par des densités gaussiennes (problème connu sous le nom d'effet leptokurtique). Ce problème se rencontrait depuis longtemps en économie, où « de telles distributions [...] ultra-étirées se manifestent fréquemment ${ }^{6}$ », et remonte au moins, pour sa première mise en évidence, à Vilfredo Pareto, dont la distribution en loi de puissance avait précisément été inventée pour modéliser ces queues de distribution étirées dans le cas de la répartition des revenus.

Pour prendre en compte ces queues de distribution non-gaussiennes, Mandelbrot proposa alors en 1962 de conserver les hypothèses iid, et de remplacer la loi de Gauss par des lois stables non gaussiennes, théoriquement bien adaptées grâce à leur comportement asymptotique parétien, à la modélisation des queues épaisses obtenues expérimentalement par les statisticiens. En d'autres termes, Mandelbrot proposa de remplacer le modèle iid-2-stable par un modèle iid- $\alpha$-stable, avec $\alpha<2$. C'était une généralisation naturelle du modèle standard dans laquelle la loi d'échelle (1) se généralisait en

$$
\Delta X \sim(\Delta t)^{1 / \alpha}
$$

L'objectif de Mandelbrot, tel qu'il le relate lui-même, était de choisir un type d'aléa plus apte à modéliser les fluctuations erratiques observées, que ne pouvaient le faire les aléas gaussiens :

6. SAMUelson, 1972, p. 31. 
«Quelle était donc l'idée centrale de mes travaux sur la finance ? L'idée, que l'on peut appeler ambiante, suivait la physique en utilisant le mouvement brownien. Elle admettait que les prix sont des fonctions continues du temps et que leurs fluctuations ne sont pas plus sévères que celles que décrit la distribution bien classique de Gauss. Mais l'examen des faits montrait le contraire : des fluctuations discontinues et des fluctuations tout à fait extrêmes. Tandis que le hasard brownien pouvait très légitimement être qualifié de "bénin ", j'ai vite dû conclure que la Bourse impliquait une toute autre forme de hasard ${ }^{7}$. »

Le réglage de la «forme » du hasard était opéré par le choix de l'exposant caractéristique $\alpha$ des lois stables. Lorsque $\alpha$ était égal à 2, on retrouvait la gaussienne et le hasard «classique », hasard bénin ou « sage », sans surprise pour les rentabilités boursières dont les fluctuations tempérées permettaient un contrôle de risque facile. Dans les autres cas $(\alpha<2)$, on se trouvait devant une nouvelle forme de hasard, plus déroutante que le hasard gaussien, appelée par Mandelbrot hasard «sauvage », dans le sens où des ruptures brutales de marché pouvaient survenir, et d'autant plus brutales que $\alpha$ s'éloignait de 2 : les fluctuations boursières devenaient tropicales, et les déluges boursiers succédaient aux emballements haussiers. Par analogie avec l'épisode biblique du déluge, Mandelbrot appela «effet Noé » cet effet dû à la variance infinie.

Mais, par cette opération intellectuelle, la propriété de stabilité par addition implicite de la gaussienne devenait tout à coup clairement apparente, et l'enjeu de l'invariance d'échelle, centre conceptuel de la géométrie fractale, surgissait comme un objet d'analyse nouveau pour la description analytique des fluctuations des marchés.

\section{b) Les difficultés expérimentales du modèle iid- $\alpha$-stable}

La validation statistique du modèle iid- $\alpha$-stable impliquait : d'une part que les valeurs obtenues pour l'exposant caractéristique $\alpha$ sur les marchés soient inférieures à 2; d'autre part, que l'exposant caractéristique soit constant quelle que soit l'échelle choisie pour l'observation (fractalité des marchés).

De manière générale, toutes les recherches entreprises sur les marchés mirent en évidence des valeurs de $\alpha$ inférieures à 2, et il n'y eut pas réellement de conflit théorique sur ce point. Le débat porta surtout sur l'hypothèse fractale, c'est-à-dire la stabilité de $\alpha$ quelle que soit l'échelle. Les contradicteurs de cette proposition cherchèrent à invalider l'hypothèse de la

7. Mandelbrot, 1997, éd. franç., p. 59 (souligné par nous). 
variance infinie en rejetant la propriété de stabilité par addition, c'est-à-dire la structure fractale des marchés.

Les premiers résultats expérimentaux furent encourageants, et confirmèrent l'hypothèse de la variance infinie et l'invariance d'échelle. Mais, assez rapidement, il apparut avec d'autres travaux que $\alpha$ présentait une tendance systématique à augmenter avec la baisse de fréquence, passant de valeurs voisines de 1,6 pour des variations quotidiennes ou hebdomadaires, à des valeurs plus proches de 1,8 pour des variations mensuelles ou trimestrielles. Cela semblait indiquer que les variations de basse fréquence étaient plus gaussiennes (moins erratiques) que les variations de haute fréquence. Entre 1970 et 1990, de nombreux auteurs mirent en évidence ce phénomène d'augmentation de $\alpha$, et en conclurent une invalidation de l'hypothèse fractale. En 1986, on lit ainsi dans un ouvrage de synthèse sur la modélisation des cours boursiers :

«Beaucoup de chercheurs ont estimé que l'hypothèse de la variance infinie n'était pas acceptable. Des études détaillées sur les variations boursières ont rejeté de manière concluante les distributions de Lévy. [...] Dix ans après son article de 1965, Fama lui-même préféra utiliser une distribution normale pour les variations mensuelles et donc abandonner les distributions stables pour les variations quotidiennes ${ }^{8}$. »

Cette apparente croissance de $\alpha$ en fonction de l'échelle constitue ce qui est appelée une «anomalie scalante», dans le sens où l'invariance d'échelle n'est pas respectée. Le passage du modèle iid-2-stable au modèle iid- $\alpha$-stable devait permettre de résoudre les anomalies de distribution. Mais une nouvelle anomalie apparaissait alors, et la question n'était pas entièrement résolue. Elle fut à l'origine d'une reconsidération de l'hypothèse de l'indépendance des variations boursières successives.

\section{c) Le problème de la mémoire et le deuxième modèle de Mandelbrot}

Dans le mouvement brownien standard, les accroissements sont indépendants (sans mémoire). La mémoire est en général mesurée par une fonction de dépendance des accroissements, dont un exposant noté $H$, qui prend ses valeurs entre 0 et 1 , caractérise la portée. Lorsque $H$ est égal à $1 / 2$, les accroissements sont indépendants (la mémoire est «courte»). Lorsque $H$ est différent de 1/2, il apparaît un phénomène de mémoire à temps long (ou corrélations à longue portée). L'hypothèse d'indépendance correspond donc à un modèle que l'on peut noter en abrégé «1/2-corrélé » (pour « accroissements à corrélation de portée réglée par l'exposant 1/2»).

8. TAYLOR, 1986, p. 46 (souligné par nous). 
La question de l'absence de dépendance (de mémoire) des variations boursières rencontrait un courant de réflexion plus ancien. Si les premiers tests d'indépendance réalisés sur les marchés avaient conclu à une absence de mémoire courte des variations boursières successives, une mémoire longue fut observée dès les années soixante sur des séries économiques et ce phénomène était décrit comme «caractéristique des fluctuations des variables économiques ${ }^{9} »$. À cause de cette dépendance longue, les cycles de basse fréquence résistaient à l'analyse spectrale.

Pour surmonter cet obstacle, Mandelbrot proposa en 1965 de généraliser le mouvement brownien standard par le mouvement brownien fractionnaire, en remplaçant la valeur $H=1 / 2$ par les autres valeurs possibles de $H$, et passant ainsi d'un modèle 1/2-corrélé des variations boursières à un modèle $H$-corrélé dans lequel la loi d'échelle (1) se généralisait en

$$
\Delta X \sim(\Delta t)^{H}
$$

Cette généralisation naturelle correspondait pour le traitement des cycles de marché, à ce qui avait été fait en 1962 pour le traitement des sauts de marché. À une variance infinie (modèle de 1962, ou « effet Noé ») répondait une mémoire infinie, que Mandelbrot appela « effet Joseph » en référence à l'épisode biblique des cycles lents de vaches grasses et de vaches maigres dans l'histoire de Joseph. La «forme » du hasard se réglait dans ce cas par le choix des valeurs de l'exposant $H$ : lorsque $H$ était égal à 0,5 , on retrouvait le hasard classique du mouvement brownien, caractérisé par de petites fluctuations autour d'une tendance. Dans les autres cas, il apparaissait des pseudo-cycles apériodiques, amples et lents, de plus en plus « apparents » à l'œil, d'une durée d'autant plus longue que $H$ se rapprochait de 1. La fractalité des marchés devenait associée à l'existence de lois d'échelles, non plus sur les sauts, mais sur les cycles.

\section{d) La validation expérimentale du modèle $H$-corrélé sur les volatilités boursières}

L'invariance par changement d'échelle, ou structure fractale du marché, fut alors cherchée sur les cycles boursiers. Si les tests statistiques de validation du modèle $H$-corrélé firent apparaître une absence de mémoire longue sur les cours eux-mêmes, les résultats furent satisfaisants sur les volatilités, où des lois d'échelles significatives apparurent nettement dans les travaux (voir exemple fig. 1, infra p. 65). L'on peut dire que, si les années 19701990 avaient été celles d'un rejet presque inconditionnel des fractales en finance, les années 1990-2000 virent le retour en force de la notion de loi d'échelle sur les fluctuations boursières.

9. GRANGER, 1966. 


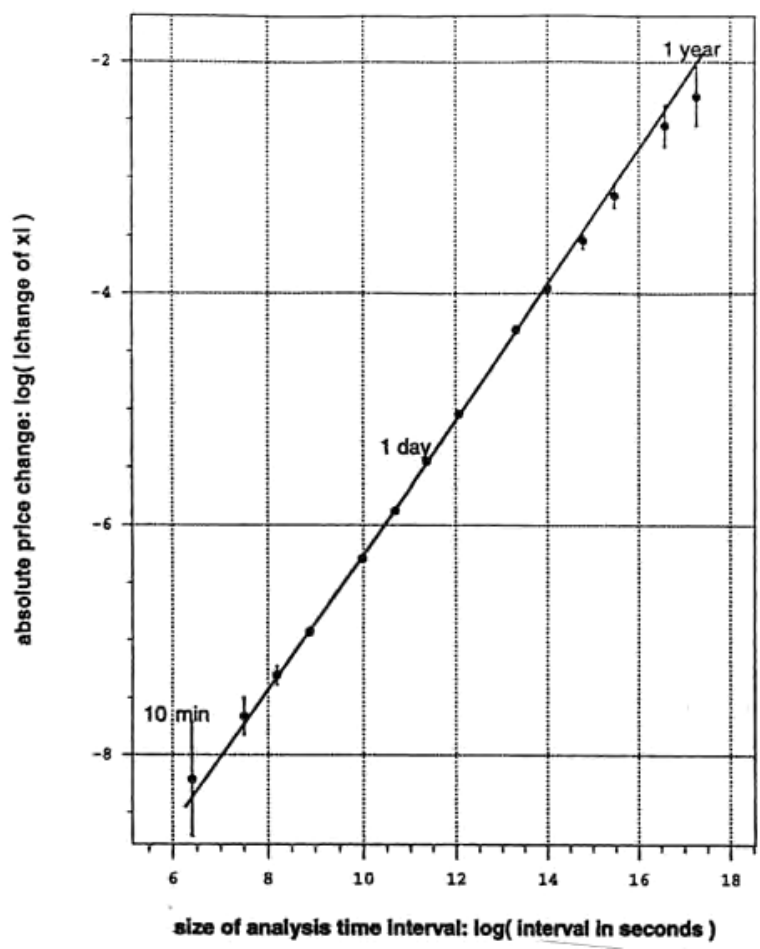

Fig. 1. - Source : MülLER et al., 1993, p. 4. Un exemple de loi d'échelle sur les variations absolues du taux de change US Dollar-Deutsche Mark

Le graphique ci-dessus représente, en coordonnées bilogarithmiques, les variations absolues des logarithmes du taux de change USD-DEM (en ordonnée) entre février 1986 et septembre 1993, soit pendant 11230 jours de bourse, en fonction de la taille de l'intervalle de temps choisi comme échelle de la variation (en abscisse). Les valeurs de l'intervalle du pas de temps varient de 10 minutes à 1 an, ce qui représente un facteur de 50 000. Les barres verticales sont les mesures d'erreurs des observations.

Si $S_{t}$ représente le taux de change USD-DEM à l'instant $t$, on pose $X_{t}=\log S_{t}$ et $\Delta X_{t, \tau}=$ $\log S_{t}-\log S_{t-\tau}$ où $\tau$ représente la largeur de la fenêtre d'observation ("pas de temps »). $\Delta X_{t, \tau}$ représente la différence des Log (taux de change) sur l'intervalle de temps $[t-\tau, t]$, et $\left|\Delta X_{t, \tau}\right|$ la valeur absolue de cette différence. On obtient la loi d'échelle

$$
\overline{|\Delta X(\tau)|} \approx \tau^{H}
$$

pour $\tau$ variant de 10 minutes et 1 an, où $\overline{|\Delta X(\tau)|}$ est la moyenne des observations $\left|\Delta X_{t, \tau}\right|$ sur la période totale.

La valeur de $H$ est de 0,59 . Les variations absolues du taux de change présentent donc une invariance d'échelle, ou encore une structure fractale, entre 10 minutes et 1 an: l'amplitude desvariations (que mesure le choix de la valeur absolue) est un phénomène que régit une loi de puissance, donc sans échelle caractéristique. Ce qui signifie qu'il n'y a pas d'intervalle de 
temps privilégié pour analyser l'amplitude des fluctuations du taux de change USD-DEM sur la zone de fractalité $[10 \mathrm{mn}, 1 \mathrm{an}]$ : sur cette zone, le marché du taux de change USD-DEM ne présente pas de différence de comportement entre les horizons de court terme et de long terme. Enfin, la valeur de $H$ est à comparer avec 0,5 pour un mouvement brownien classique $: H=0,59$ implique un écart au mouvement brownien, et correspond à l'existence d'un processus fractal à mémoire infinie au sens de Mandelbrot.

La volatilité des rentabilités boursières est une mesure de leur dispersion. Cette notion est devenue une composante primordiale des marchés d'instruments dérivés, qui se sont transformés en marchés de volatilité. Cet indicateur de variabilité entre, par ailleurs, dans la reconstitution de ce qui est appelé le «juste prix» des instruments dérivés pour les évaluations comptables des positions de marché des établissements financiers. D'où les préoccupations des instances de régulations des bourses, qui surveillent les fluctuations des niveaux de volatilité et les valeurs retenue dans les évaluation des «justes prix ». Or cette volatilité varie au cours du temps de manière déroutante et difficilement contrôlable.

L'histoire des efforts entrepris depuis vingt ans par la recherche théorique en finance pour modéliser les fluctuations erratiques de la volatilité mériterait d'être mieux connue. Rarement domaine de recherche n'a fait l'objet d'autant d'investigations et de projets d'étude, n'a reçu autant de financements privés ou publics, n'a mobilisé autant d'énergie parmi les chercheurs. Mais, les unes après les autres, les modélisations classiques de la volatilité se sont toutes trouvées confrontées au problème de la mémoire longue. Un exemple emblématique est celui de la modélisation $\mathrm{ARCH}^{10}$, introduite en 1982 par Robert Engle, courant de modélisation qui a concentré les efforts de l'industrie financière pendant plus d'une décennie ${ }^{11}$. En 1997, on pouvait lire ce commentaire sur le courant ARCH :

«Cependant, l'inflation récente de variantes du modèle de base et des terminologies : GARCH, IGARCH, EGARCH, TARCH, QTARCH, SWARCH, ACD-ARCH, révèle que cette approche semble avoir atteint ses limites, ne pas pouvoir répondre de façon adéquate à certains questions, ou ne pas permettre de reproduire certains faits stylisés ${ }^{12}$. »

Ces «faits stylisés » concernent, en particulier, la longue mémoire, ou invariance d'échelle sur la volatilité, qui est apparue vers la fin des années quatre vingts. Les tests de validation de lois d'échelle sur la volatilité ont été concluants, et la dépendance longue de la volatilité est aujourd'hui reconnue comme un fait acquis sur les marchés financiers ${ }^{13}$.

10. « Autoregressive Conditional Heteroskedasticity » : modélisation qui consiste à introduire une mémoire courte sur la variance conditionnelle. Les modélisations ARCH correspondent à une analyse multiéchelle des marchés.

11. Pour une synthèse sur les modèles ARCH en finance, voir p. ex. Bollerslev, Chou et KRONER, 1992.

12. Gouriéroux et Le Fol, 1997, p. 8 (souligné par nous).

13. Voir, p. ex., BAILLIE, 1996. 
En revanche, aucun phénomène de mémoire longue n'apparaît sur les variations de cours eux-mêmes, et la question du processus générateur des rentabilités boursières reste ouverte. Les succès rencontrés par la modélisation fractale sur le second processus, celui des volatilités boursières, laissent cependant en suspens le champ de la modélisation des cours cotés, c'est-à-dire le processus de base. La conciliation entre absence de mémoire longue sur les rentabilités et présence de mémoire longue sur les volatilités est un problème actuel de la modélisation financière. Cette question fait l'objet du troisième modèle de Mandelbrot, et d'un courant de recherche aujourd'hui très actif dans la finance théorique, celui de la mesure adéquate du temps.

LA QUESTION DU TEMPS DES MARCHÉS : DU TEMPS PHYSIQUE AU TEMPS DÉFORMÉ

Dans la modélisation des variations des cours boursiers, quelque chose résiste à la démarche usuelle, que cette démarche soit standard ou fractale, et ce «quelque chose » se trouve à l'origine de la résurgence d'une nouvelle anomalie de modélisation pour chaque résolution d'une anomalie antérieure. Examinons à nouveau la structure des modélisations financières. Le principe de base des généralisations fractales de la modélisation financière classique a été l'extension des lois d'échelle implicites de cette modélisation aux autres cas non-standard. Cela apparaît clairement lorsqu'on unifie ces modèles au moyen de la forme donnant la relation générale des lois d'échelle sur les accroissements :

$$
\Delta X \sim(\Delta t)^{H(t)}
$$

Dans les trois cas, $H(t)$ est égal à une constante : $1 / 2$ pour le modèle standard, $1 / \alpha$ dans le modèle iid- $\alpha$-stable, $H$ dans le modèle $H$-corrélé. Ces trois modélisations fractales sont unifractales dans la mesure où la valeur de $H$ est unique et définit complètement la structure fractale du marché. La dimension fractale est unique et les trajectoires boursières possèdent la même irrégularité à tout instant.

La non-résolution des anomalies au moyen de ces modèles a conduit Mandelbrot à concevoir une troisième possibilité de description analytique, apparue très tôt (en 1967) dans l'histoire de la modélisation financière mais laissée en jachère pendant une trentaine d'années : la possibilité pour $H(t)$ de varier selon $t$, c'est-à-dire introduire un cadre multifractal. Dans ce cas, les trajectoires boursières présentent une irrégularité variable qui dépend de chaque instant, la dimension fractale du marché n'est pas constante, et 
l'objectif d'obtenir un invariant dans la modélisation financière n'est pasatteint. C'est alors qu'apparut la question de la mesure du temps :

« Nous en arrivons maintenant à une idée cruciale. Cessons donc d'exprimer la variabilité du prix en termes d'un exposant variable basé sur le temps d'horloge. On peut tout aussi bien [...] concevoir une variabilité qui aurait un exposant constant, mais se produirait dans un « temps boursier » qui coulerait à un rythme très irrégulier. [...] La Bourse n'est pas esclave du temps des horloges physiques; tout au contraire, son activité de cesse de s'accélérer ("se réchauffer") et de se ralentir ("se refroidir") ${ }^{14}$."

L'invariance recherchée est alors le résultat d'une compréhension globale des fluctuations des prix et des fluctuations du temps, qui unifie ces deux dimensions de variabilité dans un cadre géométrique fractal dans lequel le temps boursier n'est plus indépendant de l'action des opérateurs : en temps multifractal, le marché présente une structure unifractale, c'est-àdire une dimension fractale constante.

Des notions jusqu'ici restées en dehors de la modélisation financière, que des codes langagiers communautairement acceptés par les professionnels désignent par «profondeur» du marché, «poids» de l'échange, «température » de la bourse, acquièrent alors une pertinence nouvelle. L'activité des opérateurs agit comme une pondération des durées, en «pliant » l'espace des cotations, en déformant le temps calendaire physique exogène du marché, en temps intrinsèque endogène au marché. Les professionnels des marchés disent souvent que la valeur d'un prix coté (et donc la pertinence de la mesure) n'est pas la même selon que ce prix correspond à une transaction de cinq cent mille titres ou de cinq titres. Cette intuition des professionnels trouve une traduction scientifique dans les modèles à temps déformé. On peut dire en conclusion que, sur les marchés financiers, la durée du temps n'est pas égale à la valeur du temps.

Christian WaLter (janvier 2001).

14. Mandelbrot, 1997, éd. franç., p. 185. 


\section{LISTE DES RÉFÉRENCES}

BAILlie (Richard), 1996, «Long memory processes and fractional integration in econometrics », Journal of Econometrics, vol. LXXIII, p 5-59.

Bâle, 1994, Risk management guidelines for derivatives, Basle Committee on Banking Supervision, juillet.

Bâle, 1996, Amendment to the capital accord to incorporate market risks, Basle Committee on Banking Supervision, janvier.

Bâle, 1998, Framework for supervisory information about derivatives and trading activities, Joint Report by the Basle Committee on Banking Supervision and the Technical Committee of the Iosco, septembre.

Bâle, 1999, Trading and derivatives disclosures of banks and securities firms, Joint Report by the Basle Committee on Banking Supervision and the Technical Committee of the Iosco, décembre.

Bollerslev (Tim), Chou (Ray) et Kroner (Kenneth), 1992, « ARCH modeling in finance. A review of the theory and empirical Evidence », Journal of Econometrics, vol. LII, 1-2, p. 5-59.

Campbell (John Y.), Lo (Andrew) et MacKinlay (A. Craig), 1997, The Econometrics of financial markets, Princeton, NJ, Princeton University Press.

Gouriéroux (Christian) et LE Fol (Gaëlle), 1997, «Volatilités et mesures du risque », Journal de la Société de statistique de Paris, t. CXXXVIII, 4, p. 7-32.

Granger (Clive W.), 1966, "The typical spectral shape of an economic variable », Econometrica, vol. XXXIV, p. 150-161.

GuÉGAn (Dominique), 1994, Séries chronologiques non-linéaires à temps discret, Paris, Economica.

Iosco, 1994, Operational and financial risk management control mechanisms for other-the-counter derivatives activities of regulated securities firms, Technical Committee of International Organization of Securities Commissions, juillet.

Mandelbrot (Benoît), 1997, Fractals and scaling in finance, New York, Springer, et éd. franç. abrégée, Fractales, hasard et finance (1959-1997), Paris, Flammarion.

Müller (Ulrich), Dacorogna (Michel), Dave (R.), Pictet (Olivier), Olsen (Richard) et WARD (J.), 1993, «Fractals and intrinsic time. A challenge to econometricians », Zurich, Olsen \& Associates Research Group, UAM 199308-16.

Samuelson (Paul A.), 1972, L'Économique, Paris, Armand Colin.

TAYLOR (Stephen), 1986, Modelling financial time series, New York, NJ, John Wiley \& Sons.

Walter (Christian), 1996, «Une histoire du concept d'efficience sur les marchés financiers », Annales. Histoire, sciences sociales, juill.-août, 4, p. 873-905.

WALTER (C.), 2001, « La recherche de lois d'échelle sur les variations boursières », chap. XIII, in Patrice ABry, Paulo Gonçalves et Jacques LÉvy-Venel, Fractales et lois d'échelle, Paris, Hermès, à paraître. 